\title{
Sport nach Knie- und Hüftendoprothetik
}

\author{
Friedrich Boettner, Maximilian F. Kasparek, Kilian Rueckl, Christian Liebau
}

Mit der Einführung neuer Materialien in die Hüft- und Knieendoprothetik sowie der Verbesserung der Prothesenverankerung im Knochen scheinen die Voraussetzungen für eine stärkere Belastung künstlicher Endoprothesen erfüllt. Die Empfehlungen hinsichtlich der sportlichen Betätigung nach Implantation einer Knie- oder Hüftprothese sind in den letzten 20 Jahren jedoch den implantattechnischen Verbesserungen nicht angepasst worden. Gerade in der Hüftendoprothetik erlaubt die heute bevorzugte Verwendung von unzementierten Implantaten sowie der Einzug von hoch vernetztem Polyethylen einen deutlich aktiveren Lebenswandel, ohne dass erhöhte Lockerungsraten und Komplikationen befürchtet werden müssen. Die hier vorgestellte Veröffentlichung fasst den gegenwärtigen Kenntnisstand aus der Literatur zusammen.

\section{Einleitung}

Die Ausübung von Sport hat einen positiven Einfluss auf die Lebensqualität, und Sport hat für viele Menschen heute bis ins hohe Alter eine zunehmende Bedeutung. Es ist davon auszugehen, dass der Anteil an Patienten, die nach einer Endoprothesenoperation sportlich aktiv sein wollen, zunehmen wird [1]. Aufgrund moderner, langlebiger Materialien und Implantate erhalten zunehmend immer jüngere Patienten, die unter einer degenerativen Erkrankung der Hüfte oder des Kniegelenks leiden, ein künstliches Gelenk. Dieses jüngere und oftmals sportlich aktive Patientenkollektiv hat aber auch eine höhere Erwartungshaltung und wünscht in der Regel nach erfolgreicher Operation eine Rückkehr in den Sport [2].

Nach einer Prothesenoperation zeigt sich in der Regel auch wieder eine stärkere sportliche Belastung, auch wenn es sich hier in der Regel um „Low-Impact“-Sportarten handelt. [3] [4]. Faktoren wie der präoperative Aktivitätslevel, männliches Geschlecht, Alter und BMI haben einen Einfluss auf die sportliche Aktivität nach der Operation. Generell wird heute bis ins gehobene Alter Sport ausgeübt, da ältere Patienten aufgrund ihrer Pensionierung oft auch wieder mehr Zeit für diese Aktivitäten zur Verfügung haben und Sport bis ins fortgeschrittene Alter heute gesellschaftlich stärker akzeptiert und gefördert wird [5].

Dabei gehört zu einer hohen Patientenzufriedenheit heute einerseits ein schmerzfreies Gelenk und die uneingeschränkte Ausübung von Alltagsaktivitäten, aber andererseits auch die individuelle Möglichkeit, Sport auszuüben [6]. Dies stellt den operativ tätigen Orthopäden und Unfallchirurgen vor die Frage, in welchem Umfang Patienten nach der Operation sportlich aktiv werden können und ob das sportliche Aktivitätsniveau der der Implantation vorausgegangenen Jahre auch nach einer Endoprothese noch sinnvoll ist.

In der Vergangenheit ist man davon ausgegangen, dass die physische Aktivität eines Patienten einen starken Einfluss auf das Abriebverhalten der Prothesen hat [7]. Insofern überrascht es auch wenig, dass die großen Fachgesellschaften wie die Hip Society und die Knee Society in ihren Richtlinien die Teilnahme an sogenannten High-Impact-Sportarten wie Handball, Basketball und Joggen nach der Implantation einer künstlichen Hüft- und Knieprothese ablehnen [8] [9] [10].

Neuere Studien haben jedoch gezeigt, dass gerade in der Hüftendoprothetik bereits Standardimplantate, die hoch vernetztes Polyethylen verwenden, derart exzellente mechanische Eigenschaften haben, dass Abrieb auch unter starker Belastung nur noch als ein unwahrscheinlicher Fehlermechanismus gilt [11].

Darüber hinaus haben die Rekonstruktion der Kapsel und Außenrotatoren nach einem hinteren $\mathrm{Zu}$ gang [12], die Verwendung des direkten vorderen Zugangs [13] [14] [15] und neue größere Kopfdurchmesser die Luxationsraten deutlich gesenkt. Damit erscheint 
auch die Ausübung von Sportarten, die einen maximalen Bewegungsumfang benötigen, wie zum Beispiel Yoga, durchaus möglich. Wesentlich weniger klar ist, inwieweit das hoch vernetzte Polyethylen eine stärkere Belastung nach einer Knieprothese ermöglichen kann. Da die stärkere Vernetzung des Polyethylens insgesamt das Material etwas brüchiger macht und die „fracture toughness" reduziert [16] wird kontrovers diskutiert, ob die Verwendung hochvernetzten Polethylens auch in der Knieendoprothetik sinnvoll ist [17] [18].

Es ist davon auszugehen, dass bei der Artikulation des runden Femurkondylus und des relativ flachen Polyethyleneinsatzes aufgrund der geringen Kontaktflächen im Vergleich zum Kugelgelenk an der Hüfte deutlich höhere Belastungsspitzen im künstlichen Kniegelenk auftreten [17]. Deshalb wurden in der Vergangenheit Sportarten wie Joggen, die mit hohen Spitzenbelastungen und unkontrollierbaren Scherkräften einhergehen, nicht empfohlen [19]. Dies dürfte auch der Hintergrund dafür sein, dass bei Hüftprothesen grundsätzlich höhere Aktivitätslevel erlaubt werden als bei Knieprothesen [8].

\section{Primäre Hüftendoprothese}

In der Praxis sind die Empfehlungen hinsichtlich Sport sehr von dem betreuenden Orthopäden abhängig und weisen eine große Bandbreite auf. So ist in der Literatur der ärztliche Rat zur Vorsicht einer der Hauptgründe für eine reduzierte Sportausübung bei Patienten nach der Operation [24]. Dies kann sicher auf die in den Anfängen der Endoprothetik hohen Lockerungsraten bei jungen und aktiven Patienten zurückgeführt werden ( $\mathbf{A} \mathbf{A b} \mathbf{b}$. 1), die bis heute noch die Empfehlungen der Operateure beeinflussen [25]. Ein guter Meinungsspiegel ist die von Swanson, Schmalzried und Dorey [8] veröffentlichte Umfrage unter Mitgliedern der amerikanischen Hip and Knee Society. Im Rahmen eines Fragebogens zu postoperativ empfohlenen Sportarten zeigte sich, dass 95\% der orthopädischen Chirurgen die Teilnahme an „Low-Impact“-Sportarten (Radfahren, Golf) empfehlen, aber von „High-Impact“Sportarten abraten. Interessanterweise zeigte sich, dass High-Volume-Chirurgen grundsätzlich liberalere Empfehlungen zur Sportausübung geben.

Bei den im Simulator beobachteten geringen Abriebraten von hochvernetztem Polyethylen ist aber nicht davon auszugehen, dass durch eine sportliche Betätigung das Risiko für eine abriebinduzierte Lockerung erheblich ansteigt [20]. Auch klinische Ergebnisse von Garvin [21] zeigen, dass junge Patienten mit einem Alter von bis zu 50 Jahren nach der Verwendung hochvernetzten Polyethylens auch nach 9 Jahren nur minimalen Abrieb zeigen.

Insofern scheinen gerade Aktivitäten, die zu einer gleichmäßigen, wenn auch intensiven Belastung der Prothese führen (langsames Joggen, Langlauf, Fahrradfahren,

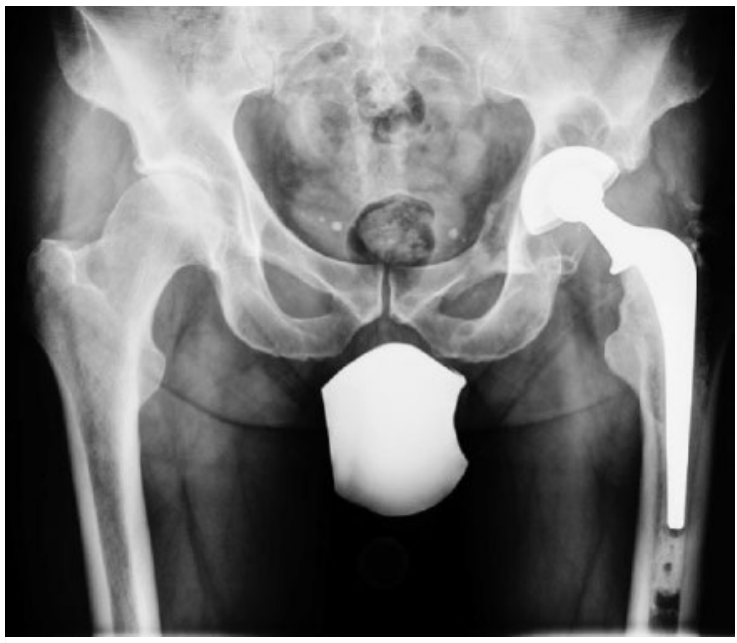

- Abb. 1 Röntgenaufnahme 14 Jahre nach Implantation einer Hüftprothese mit unvernetztem Polyethylen. Die Aufnahme zeigt ausgedehnte Osteolysen nach Plastikabrieb. Der Patient war zum Zeitpunkt der Hüftprothesenimplantation 42 Jahre alt und blieb auch nach der Operation sportlich aktiv.

Ski-Langlauf etc.), eher unproblematisch zu sein. Wobei man anmerken muss, dass nur ungefähr $4 \%$ der Patienten nach einer Hüftprothese wirklich regelmäßig joggen gehen [22]. Als häufigsten Grund, diese Sportarten nicht mehr auszuüben, geben Patienten die Angst vor Verletzungen und Stürzen an.

Unproblematisch erscheinen auch Sportarten, bei denen der direkte Kontakt zum Gegner ausgeschlossen werden kann und somit unkontrollierte Bewegungen vermieden werden (Minimalkontakt-Karate, Basketball ohne Wettbewerbscharakter etc.). Wenig problematisch erscheinen auch Sportarten, bei denen ein geübter Patient zu jedem Zeitpunkt die Kontrolle über das Hüftgelenk behält. So empfehlen wir, beim alpinen Skifahren Pisten unter dem Schwierigkeitsniveau vor der Operation auszuwählen, die es dem erfahrenen Skifahrer ermöglichen, Stürze zu vermeiden. Der Einfluss von Skifahren auf eine Hüftprothese wurde von Geschwend und seinen Mitarbeitern untersucht [23]. Im Rahmen dieser Studie wurde eine Gruppe von Patienten, die den alpinen Skilauf weiter betrieben, mit einer inaktiven Gruppe ohne Skifahren verglichen. Es zeigte sich, dass Skifahren im Rahmen dieser Studie keinen negativen Einfluss auf die Überlebensrate einer Hüftprothese hatte.

Geht der gewünschte Sport mit einem erhöhten Luxationsrisiko einher, so kann gegebenenfalls ein Operationszugang ausgewählt werden, der mit einem geringeren Luxationsrisiko einhergeht. So ist davon auszugehen, dass Sportarten, die eine tiefe Hüftbeugung erfordern, wie zum Beispiel Rudern, Beinpressen, Buckelpisten-Skifahren, sowie gewisse Yogaübungen, sicherer nach einem direkten vorderen oder anterolateralen Zugang ausgeübt werden können, als dies mit einem hinteren Zugang der Fall wäre. 
Nicht nur durch den Zugang allein kann die Stabilität des Hüftgelenkes in gewisse Bewegungsrichtungen verbessert werden. Die Einführung von Pfannen mit einer tripolaren Gelenkpaarung (ADM, Stryker, Mahwah, NJ, USA/ Active Articulation $^{\mathrm{TM}}$ Dual Mobility Hip System, Biomet, Warsaw, IN, USA) können unter Umständen unabhängig vom Zugang auch extreme Gelenkstellungen, wie sie im Rahmen von Yoga und Ballett notwendig sind, ermöglichen. Es erscheint den Autoren sinnvoll, darauf hinzuweisen, dass aufgrund der relativ unklaren Langzeitdaten es nicht absehbar ist, ob diese neuen Gelenkpaarungen auch unter extremer mechanischer Belastung gleich gute Langzeitergebnisse erzielen, wie dies für die Standard Hüftprothesen der Fall ist. Gerade für ältere Frauen mit Interesse an Yoga oder Dehnungsübungen können diese Implantate jedoch sehr nützlich sein. Es erscheint uns sinnvoll, den Patienten darüber zu informieren, dass Implantate, die eine bessere Beweglichkeit ermöglichen (Tripolare Pfanne), wahrscheinlich nicht in dem gleichen Maße mechanisch belastet werden können, wie dies bei den Standardgleitpaarungen (Standard Keramik/Metall mit hochvernetztem Polyethylen) möglich ist.

\section{Oberflächenersatz des Hüftgelenks}

Nachdem der Oberflächenersatz zu Beginn des 21. Jahrhunderts zunächst wie die ideale Lösung für den jungen und aktiven Patienten aussah, haben die teilweise schlechten Langzeitergebnisse in den letzten fünf Jahren die anfängliche Euphorie relativ schnell verfliegen lassen. Schlechte Langzeitergebnisse wurden anfangs vor allem für spezielle Implantattypen (ASR $®$, Depuy, Warsaw, IN, USA) [26] und Frauen veröffentlicht [27]. Heute zeigt sich jedoch, dass wahrscheinlich nur junge Männer bis zum Alter von 55 Jahren, die ein relativ großes Implantat (ab einer Femurkomponentengröße von $48 \mathrm{~mm}$ ) mit optimalem Design (Birmingham Oberflächenersatz, Smith \& Nephew, Memphis, TN, USA) erhalten haben, langfristig vergleichbare Überlebensraten wie eine unzementierte Standardprothese erreichen ( $\mathbf{A b b}$. 1, $\triangleright$ Abb. 2) [28]. Dies gilt für die primäre Arthrose und die posttraumatische Arthrose [29].

Vergleicht man Patienten mit einem Oberflächenersatz und einer konventionellen Hüftprothese, so zeigt sich, dass Patienten mit einem Oberflächenersatz häufiger sportlich aktiv sind [30]. Interessanterweise unterscheiden sich die beiden Gruppen jedoch ansonsten weder in den Hüftfunktions-Scores noch in den Schmerzwerten. Banerjee und Mitarbeiter [31] zeigen, dass fast alle Patienten nach einem Oberflächenersatz wieder sportlich aktiv waren. Jedoch zeigte die Studie auch nach einem Oberflächenersatz einen Trend hin zu „Low-Impact“-Sportarten. In der genannten Studie konnte nahezu keiner der Patienten, die präoperativ „High-Impact“-Sport (zum Beispiel Handball oder Basketball) betrieben hatten, nach der Operation wieder diesem Sport nachgehen.

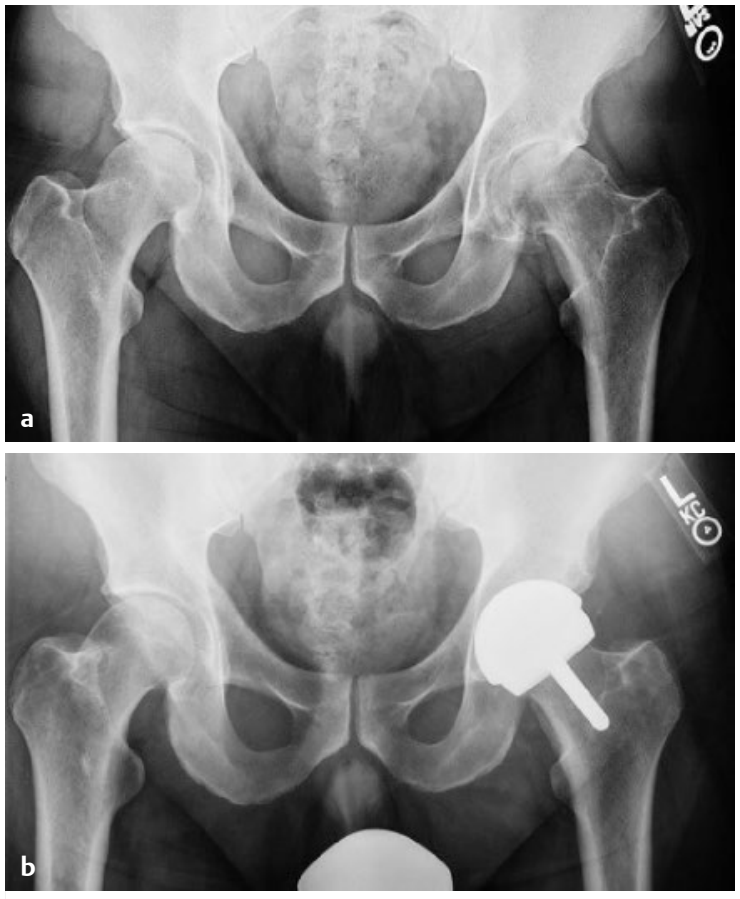

Abb. 2 Präoperatives (a) und postoperatives Röntgenbild (b) eines Mixed-Martial-Arts-Kämpfers, der im Alter von 45 Jahren einen Oberflächenersatz erhielt.

Offensichtlich ist auch, dass die Auswahl junger und aktiver männlicher Patienten für den Hüftoberflächenersatz den Prozentsatz sportlich aktiver Patienten erhöht [32]. So zeigte eine Studie von Wylde et al. [32], dass $64 \%$ der Patienten sich in den drei Jahren vor einem Oberflächenersatz aktiv sportlich betätigten, während dies nur $32 \%$ der Patienten vor einer Standard-Hüftendoprothese taten. Während in dieser englischen Patientengruppe die Wahrscheinlichkeit einer sportlichen Betätigung altersunabhängig für Männer 1,8-mal höher war als für Frauen, zeigte sich hinsichtlich des Prozentsatzes der Patienten, die nach der Operation zu ihrem präoperativen Sport zurückkehrten, kein Unterschied zwischen Standardprothese und Oberflächenersatz.

In den USA bleibt der Oberflächenersatz heute nur noch jungen Männern vorbehalten, die „High-Impact“-Sportarten ausüben. Dabei gelten als mögliche Indikation vor allem Sportarten, die (A) eine hohe mechanische Belastung des Gelenks verursachen (wettbewerbsmäßiges Laufen, Basketball und Fußball), (B) die einen extremen Bewegungsumfang des Hüftgelenks erforderlich machen (Kampfsportarten wie Karate und Judo, Klettern, Fallschirmspringen), oder (C) Sportarten, bei denen aufgrund des Umfeldes in jedem Fall eine Luxation vermieden werden muss (Wellenreiten, Windsurfen, Extremklettern und Extremski). Es ist die Erfahrung des Erstautors, dass die Mehrzahl der Patienten nach einem Oberflächenersatz auch wieder derart extreme Sportarten ausüben können. Wir schränken aus diesem Grund die sportliche Aktivität unserer Patienten nach einem Oberflä- 


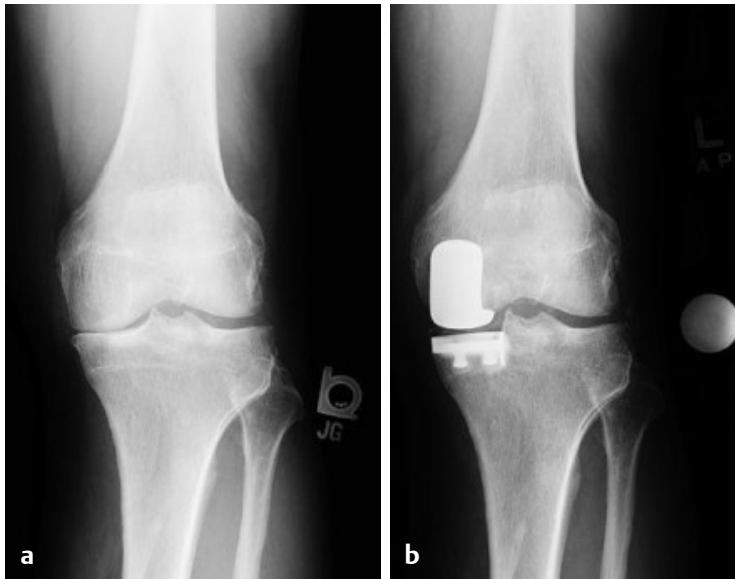

Abb. 3 Präoperatives (a) und postoperatives Röntgenbild (b) einer 65-jährigen ambitionierten Tennisspielerin. Die Patientin erhielt einen unikondylären Hemischlitten bei medialer Arthrose.

chenersatz nicht ein. Dies wird durch Studien untermauert, die gezeigt haben, dass eine stärkere Belastung bei Metall-Metallprothesen nicht unbedingt mit einer höheren Abriebrate und höherem Materialverschleiß einhergehen [33]. Aufgrund der teilweise desaströsen Ergebnisse des Oberflächenersatzess erfordert die Operation jedoch eine restriktive Indikationsstellung (Männer, Alter unter 55 Jahre, „High-Impact“-Sportarten, Implantatkopfgröße ab $48 \mathrm{~mm}$ ), einen erfahrenen Operateur, regelmäßige Nachkontrollen und die Auswahl eines Implantats mit nachgewiesener Langlebigkeit (Birmingham Oberflächenersatz, Smith \& Nephew, Memphis, TN, USA).

\section{Unikondyläre Knieendoprothese}

Unikondyläre Schlittenprothesen sind nur bei ausgewählten Patienten indiziert. In der Regel kommt die Behandlungsoption nur für Patienten infrage, die in nur einem der drei Gelenkkomparitmente degenerative Veränderungen aufweisen ( $\triangleright$ Abb. 3). Außerdem sollten eine gute Gelenkstabilität (intaktes vorderes Kreuzband), gute Beweglichkeit und relativ geringe Gelenkdeformität vorliegen.

Bei den Implantaten wird zwischen den traditionellen „Fixed Bearing“-Schlittenprothesen mit einem fixierten Plastikinlay und den „Mobile Bearing“-Schlittenprothesen mit einem mobilen Plastikinlay unterschieden. Letztere scheinen auch für schwerere Patienten (> 85 Kilogramm) eine Behandlungsoption zu sein [34].

Der Erhalt der Kreuzbänder bei dieser Operation führt zu einer normalen Gelenkkinematik und versetzt viele Patienten in die Lage, Sport zu treiben. Jedoch ist nicht klar, ob unikondyläre Schlittenprothesen den sportlichen Belastungen langfristig standhalten können.

Einige Operateure wählen aus diesem Grund Schlittenprothesen aus, die mit einem minimalen Knochenverlust bei der Implantation einhergehen und bei Lockerung aufgrund zu starker Belastung relativ unproblematisch gewechselt werden können.

Die Rehabilitation nach einer Schlittenprothese ist im Vergleich zu einer Knietotalendoprothese oft deutlich verkürzt, und die meisten Patienten erzielen ein besseres Funktionsergebnis. Patienten mit einer unikondylären Prothese beginnen häufiger und auch früher wieder mit dem Sport als Patienten nach einer Knietotalendoprothese [35] [36]. In einem vor kurzem veröffentlichten Übersichtsartikel über das Aktivitäts- und Sportniveau von Patienten mit einer unikondylären Schlittenprothese zeigte sich, dass Patienten mit einer Schlittenprothese grundsätzlich wieder regulär Sport treiben, aber das präoperative Aktivitätsniveau in der Regel nicht mehr erreichen. Auch für die Schlittenprothesen zeigt sich ein Trend zu „Lower-Impact“-Sportarten wie Schwimmen oder Radfahren [37] [38].

\section{Totale Knieendoprothese}

In der Knieendoprothetik wird je nach Erhalt des hinteren Kreuzbandes zwischen einer „Cruciate Retaining“- und einer „Posterior Stabilized“-Prothese unterschieden. Beiden Designs ist gemein, dass zur Verbesserung der Beugefähigkeit die Femurkomponente auf der Tibiaoberfläche nach hinten rollt. Dies soll bei der „Cruciate Retaining“-Knieprothese durch das hintere Kreuzband vergleichbar zur normalen Gelenkkinematik erfolgen, während bei der „Posterior stabilized“-Prothese der Kontakt zum tibialen Zapfen die Rückwärtsbewegung auslöst.

Bei beiden Designs artikuliert der runde Femurkondylus mit der flachen Tibiaoberfläche, und somit führen die relativ geringen Kontaktflächen zu erheblichen Belastungsspitzen im Polyethylen. Da hochvernetztes Polyethylen wesentlich härter ist, wird die uneingeschränkte Nutzung dieses Materials in der Knieendoprothetik aufgrund der eventuell erhöhten Brüchigkeit (geringere „fracture toughness “) immer noch kontrovers diskutiert. Während in der Hüftendoprothetik deutlich höhere Belastungen wahrscheinlich ohne langfristige Konsequenzen toleriert werden, kann davon bei einer totalen Knieprothese nicht sicher ausgegangen werden. In einer Arbeit von Lachiewicz [39] zeigten sich bei der klinischen Anwendung von hochvernetztem Polyethylen in der Knieendoprothetik weder Vorteile noch Nachteile.

Darüber hinaus führen viele sportliche Aktivitäten zu einer erheblichen Belastung des Quadrizeps und der Patella. Gerade bei sportlich aktiven Patienten sollte deswegen die Indikation zu einem Patellarückflächenersatz mit Vorsicht gestellt werden.

Anders als bei den oben genannten Standardimplantaten können durch die Verwendung einer Mobil-Bearing-Gleitpaarung oder durch ein Medial- oder Lateral-Pivote-De- 
sign Belastungsspitzen im Polyethylen vermieden werden. Theoretisch sollten diese Implantate aufgrund ihres Kugelgelenkcharakters auch höhere Belastungsspitzen aushalten können, jedoch ist dies nicht hinreichend untersucht.

Bei Insuffizienz der Seitenbänder oder stärkerem Knochensubstanzverlust kann auch die Implantation einer stärker geführten Prothese wie einer „Constrained“- oder Scharnierprothese notwendig werden. Diese Implantate sind vor allem bei Patienten mit einer ausgeprägten präoperativen Gelenkdeformität oder -instabilität beziehungsweise bei einer Prothesenwechseloperation indiziert. Aufgrund einer stärkeren Kopplung übertragen sie in ausgeprägterem Maß Stress auf das Implantat-Knochen-Interface beziehungsweise die Gleitpaarung selbst. Von einer stärkeren Belastung der Prothese sollte aus diesem Grund in jedem Fall Abstand genommen werden. Gerade für Patienten mit einer Scharnierprothese sind nur minimale sportliche Belastungen wie zum Beispiel das Golfspielen unter Verwendung eines Golfcarts, das leichte Fahrradfahren und Wandern im Flachland (vermeide Berg- und Treppauf/-ab), sowie Schwimmen zu empfehlen.

In Bezug auf spezielle Sportarten zeigt sich bei Golfern, dass die Implantation einer totalen Knieprothese zu einer sehr guten Schmerzreduktion führte und sie den Sport besser genießen konnten. Jedoch verwendeten mehr Patienten ein Golfcart nach der Operation [40].

Dass auch mit einer totalen Knieendoprothese ein sehr hohes Sportniveau erreicht werden kann, berichtete Michael Mont [41]. In seiner Studie an 33 Tennisspielern, die eine totale Knieprothese erhielten, spielten nach 7 Jahren alle Patienten wieder regelmäßig durchschnittlich $3 x$ wöchentlich Tennis. Es konnte auch gezeigt werden, dass „High-Impact“-Sportarten wie Tennis oder Basketball keinen negativen Einfluss auf das Überleben einer totalen Knieendoprothese haben und eine Prothese bei einzelnen Patienten durchaus ein hohes Aktivitätslevel ermöglicht [42].

Bradbury [43] beschrieb in einem Kollektiv von 160 Patienten, die 208 Knieprothesen erhielten, dass $91 \%$ der Patienten, die vor der Operation eine „Low-Impact“-Sportart wie Bowling betrieben, diese auch nach der Operation wieder ausübten. Im Gegensatz dazu gingen jedoch nur $20 \%$ der Patienten, die vor der Operation einen „High-Impact“-Sport, wie zum Beispiel Tennis ausübten, diesem Sport auch nach der Operation wieder regelmäßig nach. Insgesamt waren in dieser Studie $77 \%$ der Patienten wieder sportlich aktiv. Abschließend kann man feststellen, dass eine Rückkehr in den Sport grundsätzlich möglich ist. Wobei nach wie vor Patienten in der Regel Sportarten treiben, die bereits vor der Operation ausgeübt wurden. Das Erlernen einer neuen, motorisch anspruchsvollen Sportart oder einer Sportart mit einem erhöhten Verletzungsrisiko ist jedoch nicht zu empfehlen.

\section{Zusammenfassung}

Dem Endoprothesenoperateur steht heute eine Vielzahl an Implantaten zur Verfügung, die es ihm ermöglichen, Implantat und Fixierung individuell den Bedürfnissen des Patienten anzupassen. Dabei sollte es in der Regel möglich sein, dem Patienten einen aktiven Lebenswandel auch nach einer künstlichen Gelenkprothese zu ermöglichen. Entscheidend ist es bereits präoperativ abzuklären, welche Sportarten der Patient auszuüben gedenkt, und dann gezielt die beste Behandlungsoption für den Patienten auszuwählen oder im individuellen Gespräch Alternativen aufzuzeigen. Den meisten Patienten wird heute auch nach einer künstlichen Gelenkprothese eine Vielzahl an sportlichen Betätigungsfeldern offenstehen.

\section{Autorinnen/Autoren}

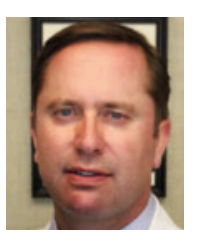

\section{Dr. Friedrich Boettner}

ist orthopädischer Chirurg und arbeitet am Hospital for Special Surgery in New York City. Sein Schwerpunkt sind die gelenkerhaltende arthroskopische Chirurgie sowie die minimalinvasive Implantation von Knie- und Hüftgelenkendoprothesen.

\section{Korrespondenzadresse}

\section{Dr. Friedrich Boettner}

Adult Reconstruction \& Joint Replacement Division Hospital for Special Surgery, 535 East 70th Street, NY 10021 New York, United States

boettnerf@hss.edu

\section{Interessenkonflikt}

Dr. Böttner ist Consultant für Smith \& Nephew, Orthodevelopment und Depuy. Dr. Boettner erhält Royalties von Orthopdevelopment. Dr. Liebau ist Consultant für DJO.

\section{Hinweis auf Zweitverwertung}

Artikel zuerst erschienen in:

Sportverl Sportschad 2017; 31: 207-212

(c) Georg Thieme Verlag KG, Stuttgart · New York ISSN 0932-0555

DOI https://doi.org/10.1055/s-0043-120880

\section{Literatur}

[1] Williams DH, Greidanus NV, Masri BA et al. Predictors of participation in sports after hip and knee arthroplasty. Clin Orthop Relat Res 2012; 470 (2): 555-561 doi:10.1007| s11999-011-2198-y

[2] Nicholls MA, Selby JB, Hartford JM. Athletic activity after total joint replacement. Orthopedics 2002; 25 (11): 1283-1287 
[3] Chatterji U, Ashworth M], Lewis PL et al. Effect of total hip arthroplasty on recreational and sporting activity. ANZ J Surg 2004; 74 (6): 446-449 doi:10.1111/j.14451433.2004.03028.x

[4] Chatterji U, Ashworth M], Lewis PL et al. Effect of total knee arthroplasty on recreational and sporting activity. ANZ J Surg 2005; 75 (6): 405-408 doi:10.1111/j.14452197.2005.03400.x

[5] Naal FD, Maffiuletti NA, Munzinger $U$ et al. Sports after hip resurfacing arthroplasty. Am J Sports Med 2007; 35 (5): 705-711 doi:10.1177/0363546506296606

[6] Bonnin M, Laurent JR, Parratte $S$ et al. Can patients really do sport after TKA? Knee Surg Sports Traumatol Arthrosc 2010; 18 (7): 853-862 doi:10.1007/s00167-009-1009-4

[7] Schmalzried TP, Shepherd EF, Dorey FJ et al. The John Charnley Award. Wear is a function of use, not time. Clin Orthop Relat Res 2000; 381: 36-46

[8] Swanson EA, Schmalzried TP, Dorey FJ. Activity recommendations after total hip and knee arthroplasty: A survey of the American Association for Hip and Knee Surgeons. J Arthroplasty 2009; 24 (6): 120-126 doi:10.1016/j. arth.2009.05.014

[9] Healy WL, lorio R, Lemos M]. Athletic activity after total knee arthroplasty. Clin Orthop Relat Res 2000; 380: 65-71

[10] Klein GR, Levine BR, Hozack W] et al. Return to athletic activity after total hip arthroplasty. Consensus guidelines based on a survey of the Hip Society and American Association of Hip and Knee Surgeons. J Arthroplasty 2007; 22 (2): 171-175 doi:10.1016/j.arth.2006.09.001

[11] Endo MM, Barbour PS, Barton DC et al. Comparative wear and wear debris under three different counterface conditions of crosslinked and non-crosslinked ultra high molecular weight polyethylene. Biomed Mater Eng 2001; 11 (1): 23-35

[12] Pellicci PM, Potter HG, Foo LF et al. MRI shows biologic restoration of posterior soft tissue repairs after THA. Clin Orthop Relat Res 2009; 467 (4): 940-945 doi:10.1007/s11999-008-0503-1

[13] Restrepo C, Parvizi J, Pour AE et al. Prospective randomized study of two surgical approaches for total hip arthroplasty. J Arthroplasty 2010; 25 (5): 671-679.e1 doi:10.1016/j. arth.2010.02.002

[14] Bergin PF, Doppelt JD, Kephart C] et al. Comparison of minimally invasive direct anterior versus posterior total hip arthroplasty based on inflammation and muscle damage markers. J Bone Joint Surg Am 2011; 93 (15): 1392-1398 doi:10.2106/jbjs.j.00557

[15] Kennon RE, Keggi JM, Wetmore RS et al. Total hip arthroplasty through a minimally invasive anterior surgical approach. J Bone Joint Surg Am 2003; 85-A (Suppl. 4): 39-48

[16] Wright TM. Polyethylene in knee arthroplasty: What is the future? Clin Orthop Relat Res 2005; 440: 141-148

[17] Sakellariou VI, Sculco P, Poultsides L et al. Highly cross-linked polyethylene may not have an advantage in total knee arthroplasty. Hss j 2013; 9 (3): 264-269 doi:10.1007| s11420-013-9352-x

[18] Renner L, Faschingbauer M, Boettner F. Is there a rationale to use highly cross-linked polyethylene in posterior-stabilized total knee arthroplasty? Ann Transl Med 2015; 3 (5): 63 doi:10.3978/j.issn.2305-5839.2015.01.17

[19] Kuster MS. Exercise recommendations after total joint replacement: a review of the current literature and proposal of scientifically based guidelines. Sports Med 2002; 32 (7): 433-445

[20] Moro T, Takatori Y, Kyomoto M et al. Wear resistance of the biocompatible phospholipid polymer-grafted highly cross-linked polyethylene liner against larger femoral head. J Orthop Res 2015; 33 (7): 1103-1110 doi:10.1002/jor.22868

[21] Garvin KL, White TC, Dusad A et al. Low wear rates seen in THAs with highly crosslinked polyethylene at 9 to 14 years in patients younger than age 50 years. Clin Orthop Relat Res 2015; 473 (12): 3829-3835 doi:10.1007/s11999-015-4422-7

[22] Abe H, Sakai T, Nishii T et al. Jogging after total hip arthroplasty. Am J Sports Med 2014; 42 (1): 131-137 doi:10.1177/0363546513506866

[23] Gschwend N, Frei T, Morscher E et al. Alpine and cross-country skiing after total hip replacement: 2 cohorts of 50 patients each, one active, the other inactive in skiing, followed for 5-10 years. Acta Orthop Scand 2000; 71 (3): 243-24 doi:10.1080/000164700317411825

[24] Huch K, Muller KA, Sturmer T et al. Sports activities 5 years after total knee or hip arthroplasty: The Ulm Osteoarthritis Study. Ann Rheum Dis 2005; 64 (12): 1715-1720 doi:10.1136/ard.2004.033266

[25] Seyler TM, Mont MA, Ragland PS et al. Sports activity after total hip and knee arthroplasty: Specific recommendations concerning tennis. Sports Med 2006; 36 (7): 571-583

[26] de Steiger RN, Hang JR, Miller LN et al. Five-year results of the ASR XL Acetabular System and the ASR Hip Resurfacing System: An analysis from the Australian Orthopaedic Association National Joint Replacement Registry. J Bone Joint Surg Am 2011; 93 (24): 2287-2293 doi:10.2106/jbjs.j.01727

[27] Bornert S, Lutzner J, Beyer F et al. Revision Rate and Patient-Reported Outcome After Hip Resurfacing Arthroplasty: A Concise Follow-Up of 1064 Cases. J Arthroplasty 2015; 30 (12): 2190-2195 doi:10.1016/j. arth.2015.06.041

[28] Smith AJ, Dieppe P, Vernon K et al. Failure rates of stemmed metal-onmetal hip replacements: Analysis of data from the National Joint Registry of England and Wales. Lancet 2012; 379: 1199-1204 doi:10.1016/s0140-6736(12)60353-5

[29] Johnson AJ, Zywiel MG, Maduekwe UI et al. Is resurfacing arthroplasty appropriate for posttraumatic osteoarthritis? Clin Orthop Relat Res 2011; 469 (6): 1567-1573 doi:10.1007| s11999-010-1655-3

[30] Zywiel MG, Marker DR, McGrath MS et al. Resurfacing matched to standard total hip arthroplasty by preoperative activity levels - a comparison of postoperative outcomes. Bull NYU Hosp Jt Dis 2009; 67 (2): 116-119

[31] Banerjee M, Bouillon B, Banerjee C et al. Sports activity after total hip resurfacing. Am J Sports Med 2010; 38 (6): 1229-1236 doi:10.1177/0363546509357609

[32] Wylde V, Blom A, Dieppe P et al. Return to sport after joint replacement. J Bone Joint Surg Br 2008; 90 (7): 920-923 doi:10.1302/0301-620x.90b7.20614

[33] Heisel C, Silva M, Skipor AK et al. The relationship between activity and ions in patients with metal-on-metal bearing hip prostheses. J Bone Joint Surg Am 2005; 87 (4): 781-787 doi:10.2106/jbjs.d.01820

[34] Pandit H, Jenkins C, Gill HS et al. Unnecessary contraindications for mobile-bearing unicompartmental knee replacement. J Bone Joint Surg Br 2011; 93 (5): 622-628 doi:10.1302/0301-620x.93b5.26214

[35] Hopper GP, Leach W]. Participation in sporting activities following knee replacement: Total versus unicompartmental. Knee Surg Sports Traumatol Arthrosc 2008; 16 (10): 973-979 doi:10.1007/s00167-008-0596-9

[36] Witjes S, Gouttebarge V, Kuijer PP et al. Return to Sports and Physical Activity After Total and Unicondylar Knee Arthroplasty: A Systematic Review and Meta-Analysis. Sports Med 2016; 46 (2): 269-292 doi:10.1007/s40279-015-0421-9 
[37] Waldstein W, Kolbitsch P, Koller U et al. Sport and physical activity following unicompartmental knee arthroplasty: A systematic review. Knee Surg Sports Traumatol Arthrosc 2016. doi:10.1007/s00167-016-4167-1

[38] Naal FD, Fischer M, Preuss A et al. Return to sports and recreational activity after unicompartmental knee arthroplasty. Am J Sports Med 2007; 35 (10): 1688-1695 doi:10.1177/0363546507303562

[39] Lachiewicz PF, Soileau ES. Is There a Benefit to Highly Crosslinked Polyethylene in Posterior-stabilized Total Knee Arthroplasty? A Randomized Trial. Clin Orthop Relat Res 2016; 474 (1): 88-95 doi:10.1007/s11999-015-4241-x

[40] Jackson JD, Smith J, Shah JP et al. Golf after tota knee arthroplasty: Do patients return to walking the course? Am J Sports Med 2009; 37 (11): 2201-2204 doi:10.1177/0363546509339009

[41] Mont MA, Rajadhyaksha AD, Marxen JL et al. Tennis after total knee arthroplasty. Am J Sports Med 2002; 30 (2): 163-166
[42] Mont MA, Marker DR, Seyler TM et al. High-impact sports after total knee arthroplasty. J Arthroplasty 2008; 23 (6): 80-84 doi:10.1016/j.arth.2008.04.018

[43] Bradbury N, Borton D, Spoo G et al. Participation in sports after total knee replacement. Am J Sports Med 1998; 26 (4): 530-535

Bibliografie

DOI https://doi.org/10.1055/a-0642-8087

Sportphysio 2018; 6: 179-185

(c) Georg Thieme Verlag KG Stuttgart · New York ISSN 2196-5951 